\title{
RITA - Robotized Inspection by Thermography and Advanced processing for the inspection of aeronautical components
}

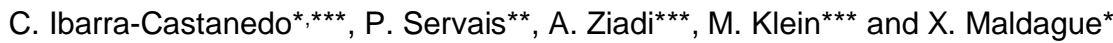

\author{
`Electrical and Computing Engineering Dept., Université Laval, Quebec City (Quebec), Canada G1K 7P4, \\ \{ibarrac,xavier.maldague\}@gel.ulaval.ca \\ *NDTPRO sprl, Le grand Enclos 18,6800 Libramont, Belgique, \{pierreservais\}@skynet.be \\ ** Visiooimage inc., 2560, rue Lapointe, Québec (QC) G1W 1A8, Canada, \{adel.ziadi, \\ matthieu.klein\}@visiooimage.com
}

\begin{abstract}
Active thermography is often performed on a static configuration where all elements of the thermographic system, i.e. the infrared camera, the energy source and the inspected object, are standing still with respect to each other. This is very useful for the application of signal processing techniques in order to improve defect detection and characterisation. Under this configuration, the large surfaces typical of some aeronautical components, are inspected in a series of static tests that at the end are assembled together on a single reconstructed mosaic image comprising the results for the entire inspected area. However, with the fast development of innovative and ever more complex-shaped parts, the alternative dynamic active thermography configuration is gaining attention. In this case, the component of interest is inspected in motion and the acquired data can be reorganized as a pseudo-static sequence, similar to classic static data, in order to perform advanced signal processing, if required. In this work, line scan thermography inspection was investigated for the assessment of an aerospace reference panel in the framework of the Canadian-Belgian collaborative project RITA (Robotized Inspection by Thermography and Advanced processing).
\end{abstract}

\section{Introduction}

Line scan thermography (LST) is a dynamic active thermography technique, which can be employed for the inspection of materials by heating a component, line-by-line, while acquiring a series of thermograms with an infrared camera. This can be done in two ways, either the thermographic head, consisting of an infrared camera and an energy source, moves along the surface while the part is motionless [1], or it may be the component that is in motion while the thermographic head stands still [2]. Two interesting variations may be adopted as well. The first one has been referred to as the thermal photocopier [3], in which the camera (separated from the thermographic head) and the inspected object are kept still with respect to each other while the energy source moves over the surface. The second one is known as flying spot imaging [4], which is similar to the photocopier principle, i.e. both the camera and the object are fixed while the thermal source moves along the specimen surface, but with the difference that there is no surface occlusion as in the thermal photocopier since heating is performed with a laser (spot or line) source. In all four cases, the thermal history for every pixel or line of pixels can be precisely tracked by controlling the heating source speed and the rate of data acquisition.

\section{Robotized line scan thermography}

An interesting way to inspect a large and/or complex-shaped aeronautical component could be for instance, by means of a robotic arm. Indeed, the use of a robot provides the possibility to program the inspection path and to control the inspection speed and acquisition rate in a precise manner. For instance, the aeronautical reference panel shown in Fig. 1a, was inspected by positioning the thermographic head over a robotic arm, as shown in Fig. 1b. The specimen is a flat $900 \times 150 \mathrm{~mm}^{2}$ monolithic CFRP panel containing 10 sections (1 to 10 as indicated) with variable number of CFRP fabric layers (progressively increasing from 6 to 22 plies). There are 3 flat-bottomed holes of different diameters (6, 8 and $10 \mathrm{~mm}$ ) per section, for a total of 30 defects located at several depths (from 0.425 to $6.09 \mathrm{~mm}$ ). The depth values are presented in Table 1, together with the diameter to depth ratios $(D / z)$.

The reference panel was positioned over a table (fixed) while the thermographic head over the robot scanned the specimen surface. Fig. 2a presents the thermal profiles over the three lines of defects (A, B, and C) obtained while performing the thermographic scan. As can be seen, the topmost defect line, line $\mathrm{C}$, which contains the shallowest defects (see Fig. 1a and Table 1), produces a thermal profile with very distinctive signatures for all the defects (C1 to C10). Even though the other two defect lines, B (center) and A (bottom) have larger defects (except for defect A5), they all show weaker signatures since they are located increasingly deeper (from left to right). A distinctive thermal contrast can be observed for only a few defects (B1, B2 and A1), and subtle indications on a few others. A reconstructed thermogram, corresponding to approximately $1 \mathrm{~s}$ after heating, is presented in Fig. $2 \mathrm{~b}$. An uncooled microbolometer 
camera (Jenoptik IRTCM 384, LWIR 7.5-14 $\mu \mathrm{m}$, 384x288 pixels) was used during data acquisition and the specimen was heated using a low power heating line lamp.

It is possible to improve defect detectability by increasing the power of the heating source. However, the robotic arm was not available at this time to perform new inspections with a higher power heating source. A series of tests with the robotic arm and a higher power source are programmed for the near future (summer 2014). Instead, a 2 axis electric actuator was employed to carry out a line scan inspection with a similar precision than the robotic arm in the case of a flat specimen as the one investigated herein. The total length of the actuator arm in the direction of inspection is $580 \mathrm{~mm}$, which limits the effective inspection length to approximately half the specimen (length $=900 \mathrm{~mm}$ ). A cooled camera (FLIR Phoenix, InSb, MWIR, 3-5 $\mu \mathrm{m}, 640 \times 512$ pixels) was employed during testing. Fig. 2c presents a thermogram reconstruction of two different scans: the left image containing the four lines with shallowest defects, and the right image corresponding to the four deepest defects lines. The two central defect lines are missing since the experimental setup at the time did not allow conducting a larger scan, hence there is a gap at the center of the image.

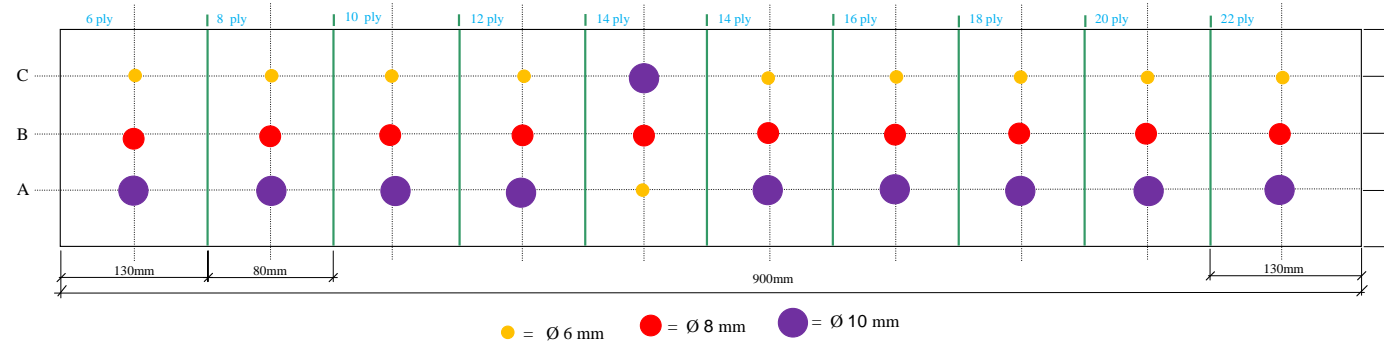

(a)

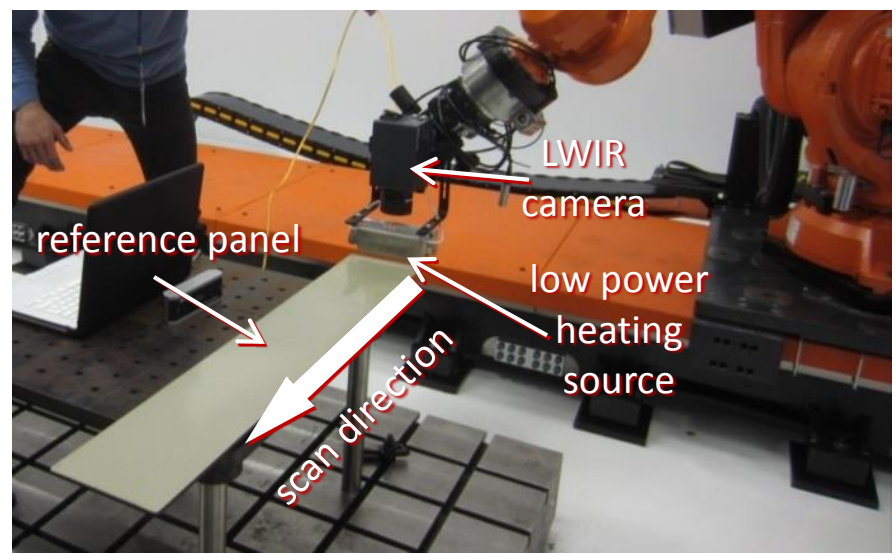

(b)

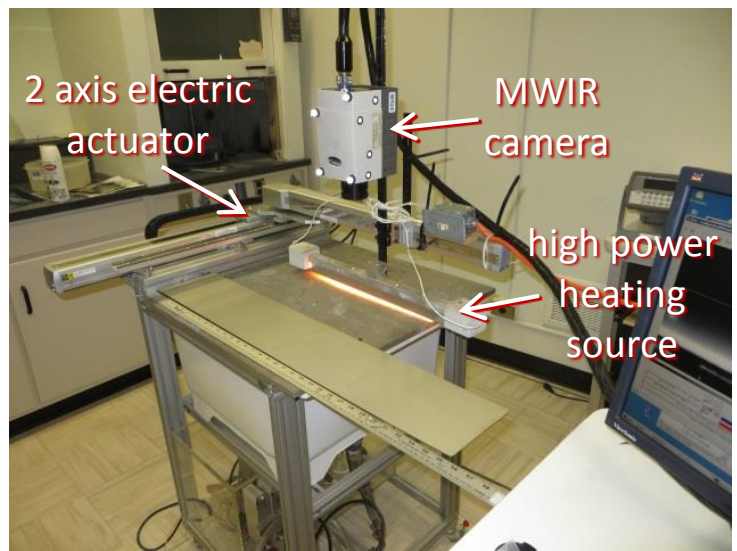

(c)

Fig. 1. (a) Defect map of the reference panel and corresponding depths; (b) robotized line scan thermography inspection with low power source; and (c) line scan system with electrical actuator with high power source.

Table 1. Depths and diameter to depth ratios corresponding to the 30 flat-bottom-holes of the reference panel.

\begin{tabular}{|c|c|c|c|c|c|c|c|c|c|c|}
\hline & \multicolumn{10}{|c|}{$\begin{array}{c}\text { Depths }[\mathrm{mm}] / \\
\text { diameter to depth ratio }(\mathrm{D} / \mathrm{z})\end{array}$} \\
\hline & $\begin{array}{c}\text { Section } \\
1 \\
\end{array}$ & $\begin{array}{c}\text { Section } \\
2 \\
\end{array}$ & $\begin{array}{c}\text { Section } \\
3 \\
\end{array}$ & $\begin{array}{c}\text { Section } \\
4 \\
\end{array}$ & $\begin{array}{c}\text { Section } \\
\mathbf{5} \\
\end{array}$ & $\begin{array}{c}\text { Section } \\
6 \\
\end{array}$ & $\begin{array}{c}\text { Section } \\
7 \\
\end{array}$ & $\begin{array}{c}\text { Section } \\
8 \\
\end{array}$ & $\begin{array}{c}\text { Section } \\
9 \\
\end{array}$ & $\begin{array}{c}\text { Section } \\
10 \\
\end{array}$ \\
\hline $\begin{array}{c}C \\
(D=6 \mathrm{~mm})\end{array}$ & $\begin{array}{c}0.88 / \\
6.8\end{array}$ & $\begin{array}{c}0.86 / \\
7.0\end{array}$ & $\begin{array}{c}0.63 / \\
9.5\end{array}$ & $\begin{array}{c}0.69 / \\
8.7\end{array}$ & $\begin{array}{c}0.94 / \\
10.6\end{array}$ & $\begin{array}{c}0.99 / \\
6.1\end{array}$ & $\begin{array}{c}0.42 / \\
14.1\end{array}$ & $\begin{array}{c}0.86 / \\
7.0\end{array}$ & $\begin{array}{c}0.54 / \\
11.0\end{array}$ & $\begin{array}{c}0.65 / \\
9.2\end{array}$ \\
\hline $\begin{array}{c}\text { B } \\
(D=8 \mathrm{~mm})\end{array}$ & $\begin{array}{c}1.2 / \\
6.5\end{array}$ & $\begin{array}{c}1.4 / \\
5.7\end{array}$ & $\begin{array}{c}1.7 / \\
4.7\end{array}$ & $\begin{array}{c}2.0 / \\
4.1\end{array}$ & $\begin{array}{c}2.2 / \\
3.6\end{array}$ & $\begin{array}{c}2.4 / \\
3.3\end{array}$ & $\begin{array}{c}2.6 / \\
3.1\end{array}$ & $\begin{array}{c}2.9 / \\
2.7\end{array}$ & $\begin{array}{c}3.1 / \\
2.6\end{array}$ & $\begin{array}{c}3.5 / \\
2.3\end{array}$ \\
\hline $\begin{array}{c}A \\
(D=10 \mathrm{~mm})\end{array}$ & $\begin{array}{c}1.5 / \\
6.6 \\
\end{array}$ & $\begin{array}{c}2.1 / \\
4.7 \\
\end{array}$ & $\begin{array}{c}2.6 / \\
3.9\end{array}$ & $\begin{array}{c}3.2 / \\
3.2\end{array}$ & $\begin{array}{c}3.5 / \\
1.7 \\
\end{array}$ & $\begin{array}{r}3.7 / \\
2.7 \\
\end{array}$ & $\begin{array}{c}4.2 / \\
2.4 \\
\end{array}$ & $\begin{array}{c}4.9 / \\
2.0 \\
\end{array}$ & $\begin{array}{c}5.5 / \\
1.8 \\
\end{array}$ & $\begin{array}{c}6.1 / \\
1.6 \\
\end{array}$ \\
\hline $\begin{array}{c}\text { No. of } \\
\text { plies }\end{array}$ & 6 & 8 & 10 & 12 & 14 & 14 & 16 & 18 & 20 & 22 \\
\hline
\end{tabular}


(a)
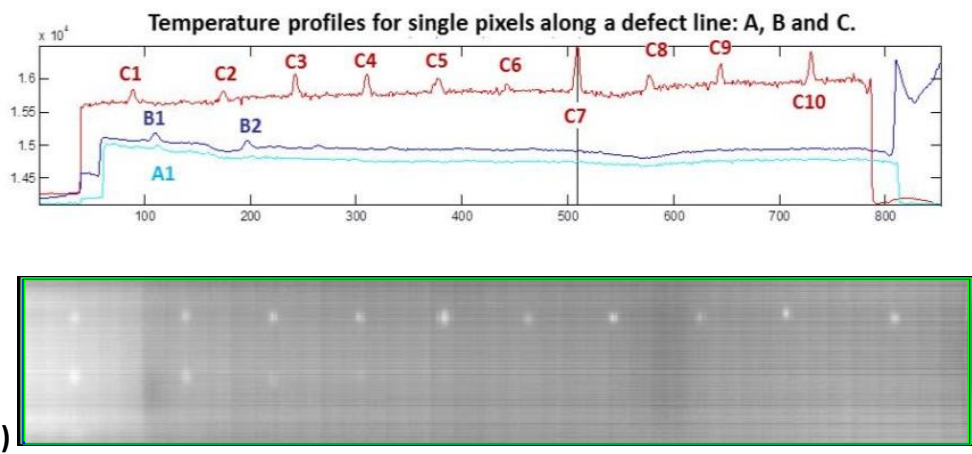

(c)

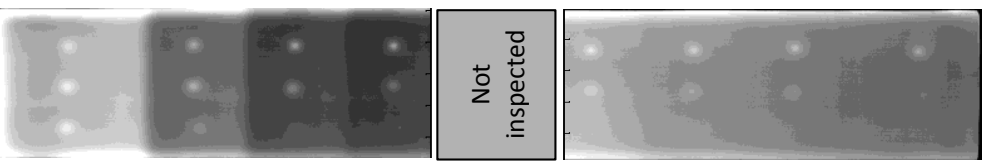

Fig. 2. (a) Raw thermal profiles for the 30 artificial defects; (b) the corresponding reconstructed thermogram from reference panel inspected at $20 \mathrm{~mm} / \mathrm{sec}$ using a low power heating source; and (c) recomposed raw thermogram from reference panel inspected at $10 \mathrm{~mm} / \mathrm{sec}$ using a high power heating source.

From the results presented in Fig. 2 it was observed that it was possible to reach defects as deep as approximately $3 \mathrm{~mm}$ (somewhere between defects A3 and A4, and between defects B9 and B10 as seen in Fig. 2c) with the correct amount of energy.

\section{Pseudo-static matrix reconstruction and adjustable parameters}

A number of parameters need to be selected during a line scan inspection. Besides the parameters common to static and dynamic configurations (camera's characteristics, field-of-view, power of the energy source, etc.), additional parameters linked to the relative movement between the inspected part, the energy source and/or the camera, need to be addressed under a dynamic configuration. The different parameters to control during a line scan inspection are:

- The amount of energy that is delivered to the specimen surface by a line scan system is controlled by:

heat source power;

size of the heating line;

scanning speed;

distance between the source and the surface.

- The spatiotemporal resolution is a function of the following parameters:

$\circ \quad$ size of the FPA matrix $\left(p_{x} \times p_{y}\right): x$ is the scanning direction and $y$ is the direction perpendicular to the scan, temporal resolution (after reconstruction) improves at larger $p_{x}$;

- field of view (FOV):

- $\quad$ distance between the camera and the inspected surface;

- camera's optical characteristics (focal length);

- optical distortions (image rectification might be needed);

- occlusions (image cropping might be needed);

- acquisition rate: better spatial resolution in the reconstructed matrix $p_{x^{\prime}}$ at higher acquisition rates (for a given scanning speed).

The reconstructed matrix is obtained by following the temporal evolution of every pixel line independently, in such a way that, a given pixel line of the original sequence $P x_{i}(t)$, is recovered frame by frame (through time $t$ ) and reallocated into a new image. For instance, the first pixel line at time $t_{1}$, that is $P x_{1}\left(t_{1}\right)$, is rearranged as the first line of the first reconstructed image corresponding to a virtual time $t^{\prime}{ }_{1}$, that is $P x^{\prime}{ }_{1}\left(t_{1}{ }_{1}\right)$. The pixel line at the same position $x_{1}$ but in the following frame (at time $t_{2}$ ), that is $P x_{1}\left(t_{2}\right)$, corresponds to the second line of the reconstructed image at the same virtual time $t^{\prime}{ }_{1}$ (the first frame), that is $P x^{\prime}{ }_{2}\left(t^{\prime}{ }_{1}\right)$, and so on. At the end, the sequence of pixel lines at position $x_{1}$ in the original sequence: $P x_{1}\left(t_{1}\right) \ldots P x_{1}\left(t_{N}\right)$, is rearranged into a single image representing the first frame at the virtual time $t^{\prime}{ }_{1}$ : $P x_{1}{ }_{1}\left(t_{1}^{\prime}{ }_{1}\right) \ldots P x_{2}{ }_{2}\left(t_{1}^{\prime}\right)$. The same is true for the rest of the pixel lines in the original sequence: 


$$
\begin{gathered}
P_{x_{1}}\left(t_{1}\right) \cdots P_{x_{1}}\left(t_{N}\right) \stackrel{\text { reconstructed }}{\longrightarrow} P_{x_{1}{ }_{1}}\left(t^{\prime}{ }_{1}\right) \cdots P_{x_{\prime_{N}}}\left(t^{\prime}{ }_{1}\right) \\
P_{x_{2}}\left(t_{1}\right) \cdots P_{x_{2}}\left(t_{N}\right) \stackrel{\text { reconstructed }}{\longrightarrow} P_{x_{1}{ }_{1}}\left(t^{\prime}{ }_{2}\right) \cdots P_{x_{N}{ }_{N}}\left(t^{\prime}{ }_{2}\right) \\
P_{x_{3}}\left(t_{1}\right) \cdots P_{x_{3}}\left(t_{N}\right) \stackrel{\text { reconstructed }}{\longrightarrow} P_{x^{\prime}{ }_{1}}\left(t^{\prime}{ }_{3}\right) \cdots P_{x_{N_{N}}{ }_{N}\left(t^{\prime}{ }_{3}\right)} \\
P_{x_{N}}\left(t_{1}\right) \cdots P_{x_{N}}\left(t_{N}\right) \stackrel{\text { reconstructed }}{\longrightarrow} P_{x_{1}{ }_{1}}\left(t^{\prime}{ }_{N}\right) \cdots P_{x_{N}{ }_{N}}\left(t^{\prime}{ }_{n}\right)
\end{gathered}
$$

Since the inspection head is moving over the inspected object, the object position is continuously shifting from one frame to the next. Unless the camera frame rate is perfectly synchronized with the scanning speed, which is rarely the case, an additional correction on shifting should be performed. This can be done by either using a calibrated target as explained by Oswald-Tranta and Sorger [5]; or by shifting correction procedure based on the interpolation between the initial and final positions of a reference pixel. In both cases, it is assumed that the camera and source move at a constant speed.

The observation time tobs (or time window), i.e. the time during which a given point (line) in the inspected object is observed at a given scanning speed $v_{x}$, can be precisely calculated by knowing the length of the FOV in the scanning direction $X$ :

$$
t_{o b s}=\frac{X}{v_{x}}
$$

The virtual acquisition rate of the reconstructed sequence $f_{\text {'rate, }}$ can then be estimated by knowing the number of pixels being scanned $p_{x}$ during the observation time from tobs:

$$
f_{\text {rate }}^{\prime}=\frac{p_{x}}{t_{o b s}}=\frac{1}{\Delta t^{\prime}}
$$

Eqs. (1) to (3) are employed to reconstruct the pseudo-static sequence from the dynamic matrix and to determine the observation time and frame rate for every pixel in the new sequence. In order to assess the impact of different acquisition parameters, a series of tests were performed on one side of the reference panel (left side in Fig. 1a) covering the five defect columns (sections 1 to 5 in Table 1) with the 5 shallowest defects for each line (A to C). This corresponds to diameter to depth ratios from 1.7 to 10.6. Some results are presented and discussed in the next section.

As an example of data reconstruction, Fig. 3a shows the thermal profile through virtual time t' of defect C1 and a sound area next to it. The profiles follow a temperature decay similar to the ones observed in a static pulsed thermography configuration. The bumps seen in the profiles at approximately $2.3 \mathrm{~s}$ are artifacts caused by lens flare due to the proximity between the camera and the heating source and the relatively high reflectivity of the specimen surface. This flare corresponding to a fixed noise in the original sequence observed around line 208 (see Fig. $3 b$ ), can be seen as a moving reflection in the reconstructed matrix visible at $t^{\prime}=2.3 \mathrm{~s}$ (see Fig. $3 \mathrm{c}$ ).

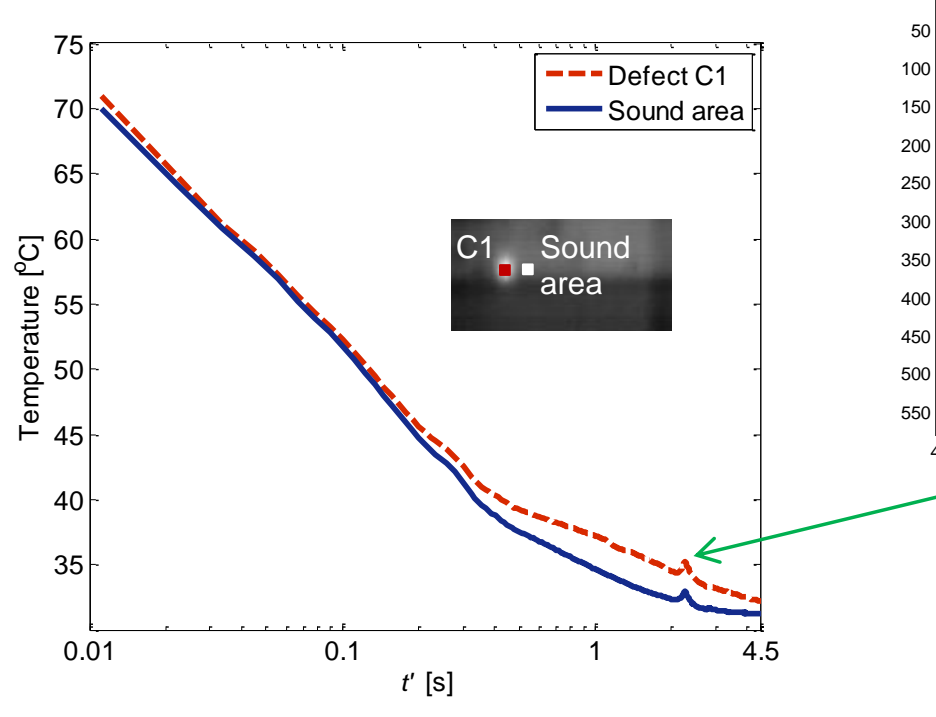

(a)

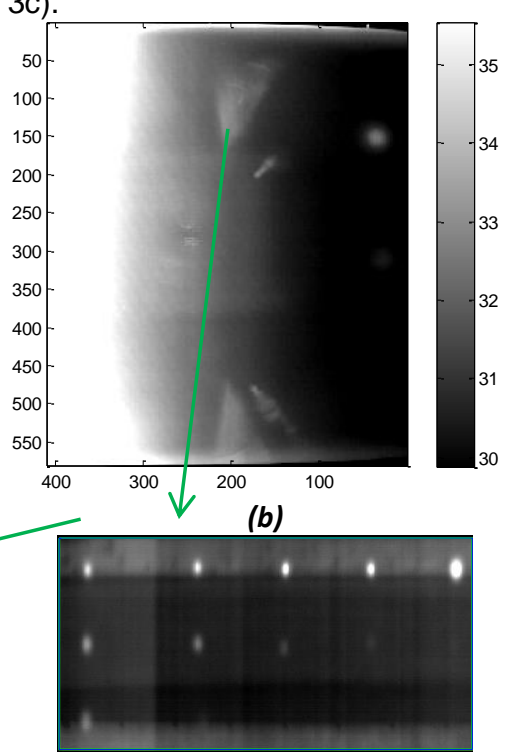

(c)

Fig. 3. (a) Thermal profile through virtual time t' of defect $C 1$ and a sound area next to it; and (b) thermogram at $t=17.7 \mathrm{~s}$ in the original matrix showing lens flare artifact around line 208; and (c) reconstructed thermogram at $t^{\prime}=2.3 \mathrm{~s}$. 


\section{Experimental results with electrical actuator}

Fig. 4 (left) presents four representative reconstructed thermograms at progressively decreasing scanning speeds (from 30 to $5 \mathrm{~mm} / \mathrm{s}$ ). The acquisition rate was adjusted according to the scanning speed in order to limit the total number of acquired images. Hence, higher acquisition rates were used (in general) for higher scanning speeds, and vice versa, as indicated. Experiments at 20 and $10 \mathrm{~mm} / \mathrm{s}$ were obtained using the same acquisition rate $\left(f_{\text {rate }}=28 \mathrm{~Hz}\right)$. The main effect was to double the number of acquired thermograms for the $10 \mathrm{~mm} / \mathrm{s}$ scan compared to the $20 \mathrm{~mm} / \mathrm{s}$ prior to pseudostatic reconstruction, and therefore, to increase the spatial resolution in the direction of the scan after reconstruction $x$ ' (see for instance the number of pixels in the thermogram and graph at $10 \mathrm{~mm} / \mathrm{s}$ with respect to the $20 \mathrm{~mm} / \mathrm{s}$ ). The total number of images before reconstruction, the resulting observation time tobs, for each experiment (shorter for faster scannings) and the time corresponding to the thermogram are also indicated.
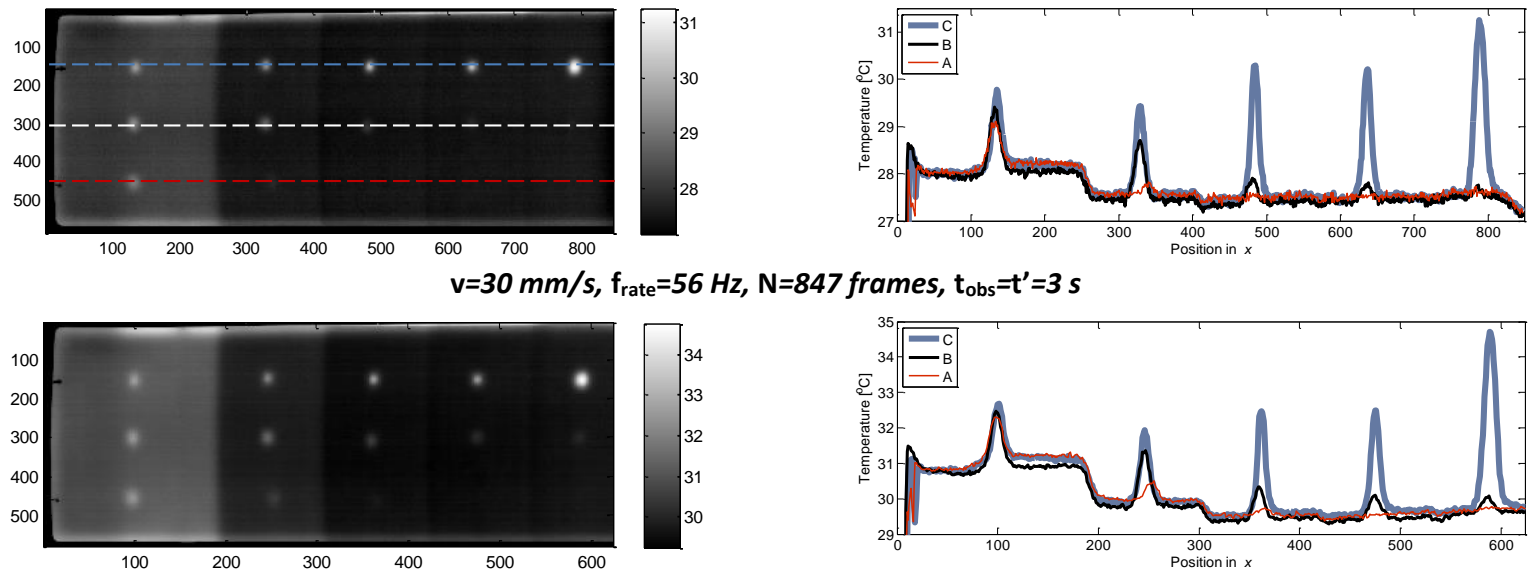

$v=20 \mathrm{~mm} / \mathrm{s}, f_{\text {rate }}=28 \mathrm{~Hz}, \mathrm{~N}=624$ frames, $t_{\text {obs }}=4.6, t^{\prime}=4.1 \mathrm{~s}$
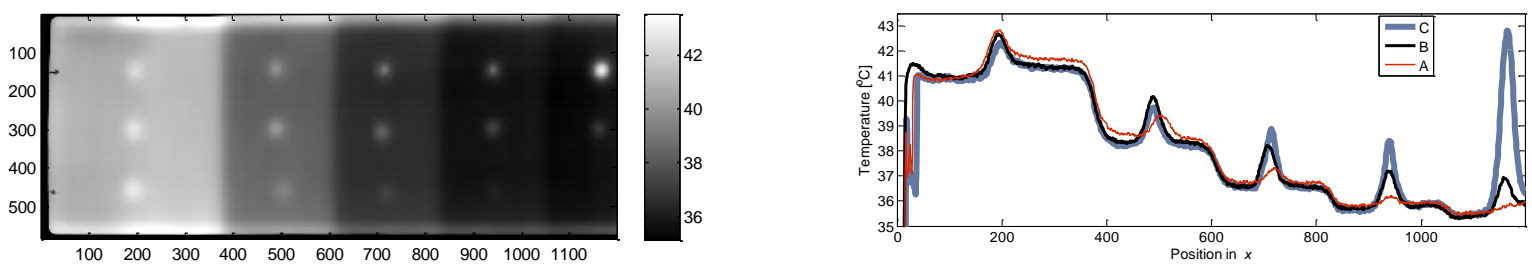

$v=10 \mathrm{~mm} / \mathrm{s}, f_{\text {rate }}=28 \mathrm{~Hz}, \mathrm{~N}=1198$ frames, $t_{\text {obs }}=9.1 \mathrm{~s}, t^{\prime}=6.7 \mathrm{~s}$
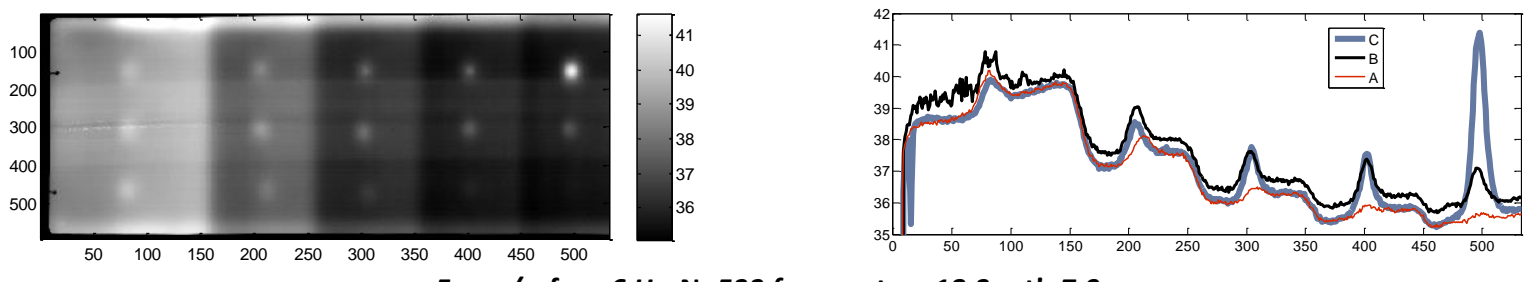

$v=5 \mathrm{~mm} / \mathrm{s}, \mathrm{f}_{\text {rate }}=6 \mathrm{~Hz}, \mathrm{~N}=533$ frames, $\mathrm{t}_{\mathrm{obs}}=18.3 \mathrm{~s}, \mathrm{t}^{\prime}=7.9 \mathrm{~s}$

Fig. 4. The impact of the scanning speed and the corresponding spatial profiles through the defects lines $C, B$, and $A$.

The spatial profiles along the three defect lines (C, B and A) are also shown in Fig. 4 (left). The bumps provide an indication of the presence of a defect with the exception of the left-most bump, which corresponds to the edge of the specimen created by border effect. The border bumps are larger for higher scanning speeds, where the specimen has less time to diffuse heat. No bumps related to the right edge are observed in these graphs since only half the panel was inspected due to limitations of the experimental setup, as explained before.

The scanning speed has an impact on the observation time as well as on the amount of energy that is transmitted to the specimen and consequently on the limiting $D / z$ that can be attaint. As can be observed from these results, defects with lower $D / z$ can be reached only when using lower scanning speed (since the observation time is increased and more energy is transferred to the specimen). The time of best detection (maximum contrast) is a function of $D / z$, therefore each defect should be analyzed independently in order to optimize defect visibility. However, for sake of simplicity, the thermograms presented in Fig. 4 were selected from the reconstructed (pseudo-static) sequence as they provided the best defect detectability for the lowest $D / z$ detected in each case. As a consequence, defects with higher $D / z$ lose some contrast in these particular images due to heat diffusion. Better detectability for these defects is of course achieved earlier in the reconstructed matrix (see Fig. 5 for comparison). 
The reconstructed thermogram at $30 \mathrm{~mm} / \mathrm{s}$ clearly shows the 5 defects in line $C$ (C1 to C5), two defects in line $B$ (B1 and B2), and only one defect in $A(A 1)$ with corresponding $D / z$ ranging from 5.7 to 10.6 . A closer look of the temperature profiles allow to identify at least two more defects in line $B$ (B3 and B4, with $D / z \geq 4.1$, respectively) and one more in line $A(A 2, D / z=4.7)$. The corresponding signal-to-noise ratios (SNR) for these three defects, as defined by:

$$
\mathrm{SNR}=20 \log _{10}\left(\frac{S_{d}-S_{S a}}{\sigma_{S a}}\right)[\mathrm{dB}]
$$

are: 18.9, 11.8 and $7.4 \mathrm{~dB}$, respectively. These SNR values correspond to the three less visible defects at this scanning speed, SNR for the other identified defects (not shown) are of course expected to be higher.

In the case of the thermogram reconstructed at $20 \mathrm{~mm} / \mathrm{s}$, all defects in lines $\mathrm{C}$ and $\mathrm{B}$ are clearly identified from their temperature profiles $(D / z \geq 3.6)$, as well as defects $A 1$ and $A 2(D / z \geq 4.7)$. Defect $A 3$ shows a slight thermal contrast corresponding to a $\mathrm{SNR}=10.1 \mathrm{~dB}$.

Slower scanning speeds of 10 and $5 \mathrm{~mm} / \mathrm{s}$, improve defect detection to $D / z \geq 3.2$, i.e. defect $A 4$ is detected $(z=3.2 \mathrm{~mm}$ for $D=10 \mathrm{~mm}$ ). The thermal profile for defect $A 5$ (the one with lowest $D / z=1.7$ ) shows only a subtle thermal contrast, too small to produce a significant SNR.

Fig. 5 presents the reconstructed thermograms corresponding to $t^{\prime}=3 \mathrm{~s}$ for the four different scanning speeds and the thermal profiles for the three line of defects ( $A, B$ and $C$ ). The top-most thermogram and graph are exactly the same as the ones presented in Fig. 4 (top) and is reproduced for comparison. The following thermograms correspond to the same time, $t^{\prime}=3 \mathrm{~s}$, but reconstructed using decreasingly slower scanning speeds.
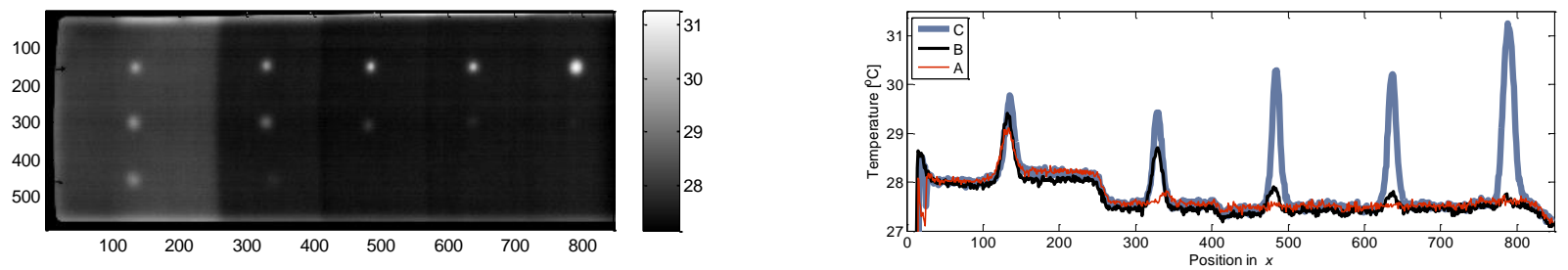

$\mathrm{v}=30 \mathrm{~mm} / \mathrm{s}, \mathrm{f}_{\text {rate }}=56 \mathrm{~Hz}, \mathrm{~N}=847$ frames, $\mathrm{t}=3 \mathrm{~s}$
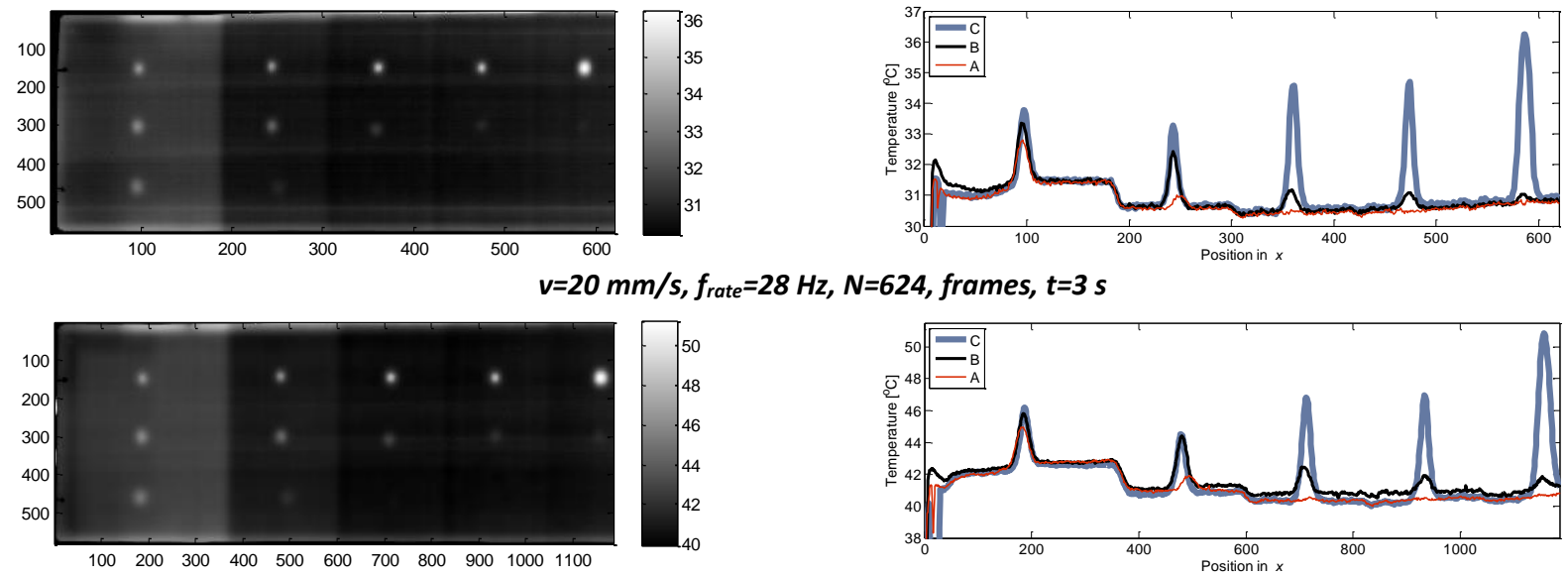

$v=10 \mathrm{~mm} / \mathrm{s}, f_{\text {rate }}=28 \mathrm{~Hz}, \mathrm{~N}=1198$ frames, $t=3 \mathrm{~s}$
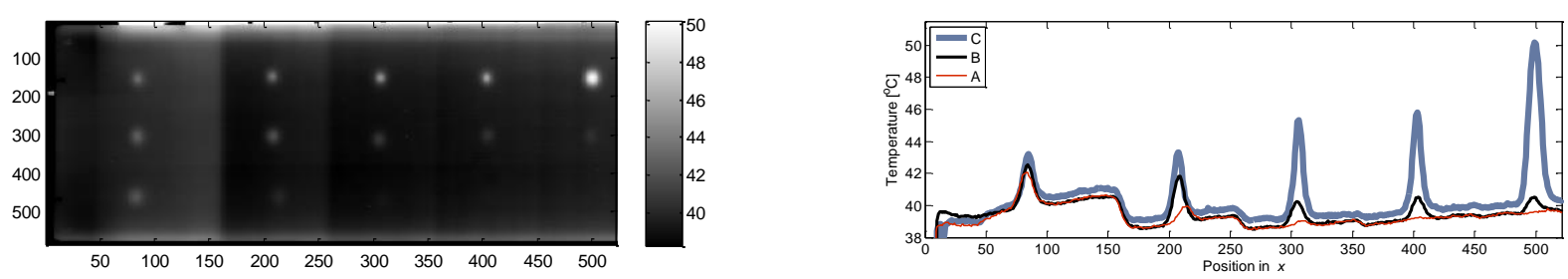

$v=5 \mathrm{~mm} / \mathrm{s}, \mathrm{f}_{\text {rate }}=6 \mathrm{~Hz}, \mathrm{~N}=533$ frames, $\mathrm{t}=3 \mathrm{~s}$

Fig. 5. The impact of the scanning speed on effective heating.

From the thermal profiles it can be observed that thermal contrast for most of the defects increases as the scanning speed slows down, see for instance defect B5 (last bump in the darkest profile), for which contrast is improved in such a way that passes from fairly detectable at $30 \mathrm{~mm} / \mathrm{s}$ (top) to clearly detectable at $5 \mathrm{~mm} / \mathrm{s}$ (bottom). This is due to the increased amount of energy delivered to the specimen surface from lateral diffusion. Since the heating line is moving slower, heat diffusion (from the line source) is greater for slower scanning (heating) speeds. The effective heat input is 
therefore greater for slower scanning speeds. This can be confirmed from the surface temperature, which progressively increases (see temperature scales in Fig. 5) as the scanning speed goes down. The last thermogram and graph were obtained from an experiment carried out at $5 \mathrm{~mm} / \mathrm{s}$ using half the power of the heating lamp to avoid over heating the sample. Hence, surface temperature is close to the one at $10 \mathrm{~mm} / \mathrm{s}$, still, a slight contrast improvement is noticeable for deeper defects (see defect B5 for instance).

The impact of the acquisition rate at a constant speed is analyzed in Fig. 6, which shows four reconstructed thermograms corresponding to the same time (after reconstruction) but using progressively decreasing acquisition rates (from 28 to $3.5 \mathrm{~Hz}$, to be compared to the top-most thermogram and graph in Fig. 4 and Fig. 5). The impact of using an insufficiently fast acquisition rate over the spatial resolution of the reconstructed thermograms is more evident in the cases where $f_{\text {rate }} \leq 7 \mathrm{~Hz}$ for a $30 \mathrm{~mm} / \mathrm{s}$ scanning speed, as observed from these results.
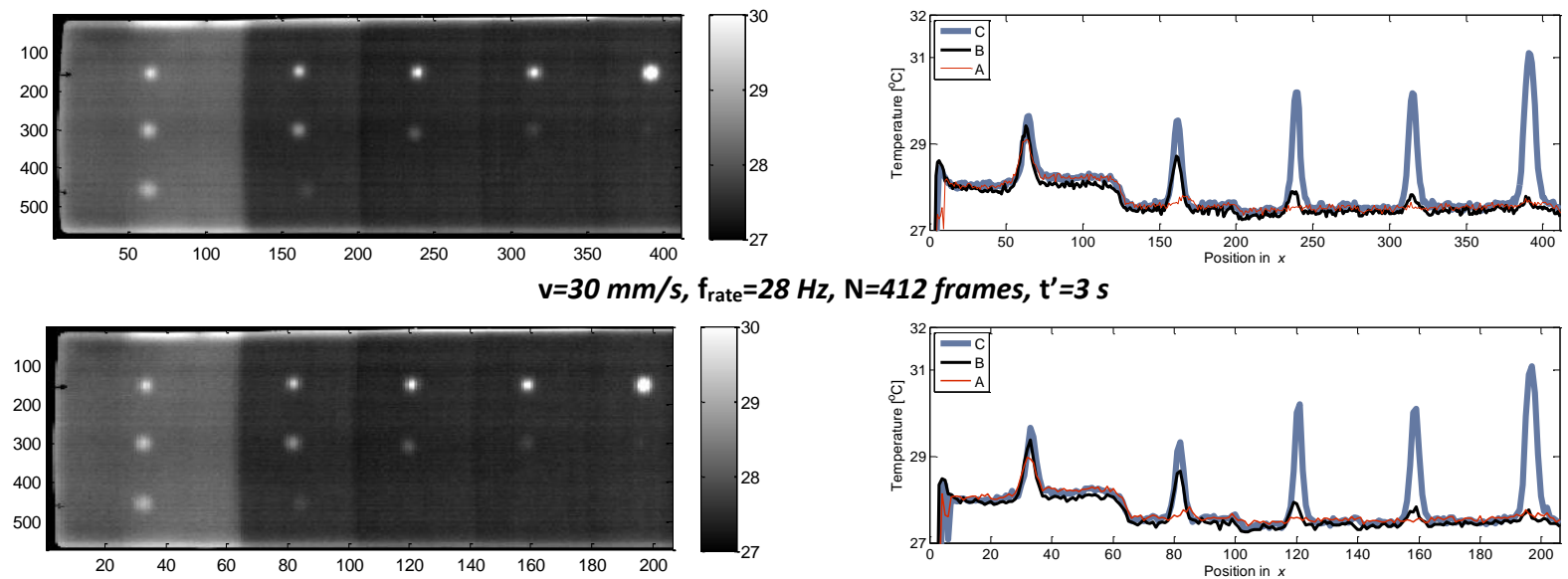

$v=30 \mathrm{~mm} / \mathrm{s}, f_{\text {rate }}=14 \mathrm{~Hz}, \mathrm{~N}=206$, frames, $t^{\prime}=3 \mathrm{~s}$
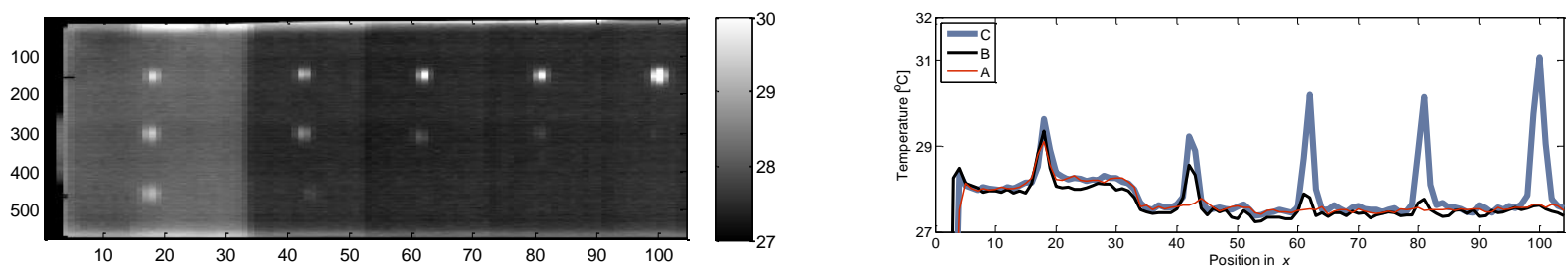

$v=30 \mathrm{~mm} / \mathrm{s}, f_{\text {rate }}=7 \mathrm{~Hz}, \mathrm{~N}=104$ frames, $t^{\prime}=3 \mathrm{~s}$
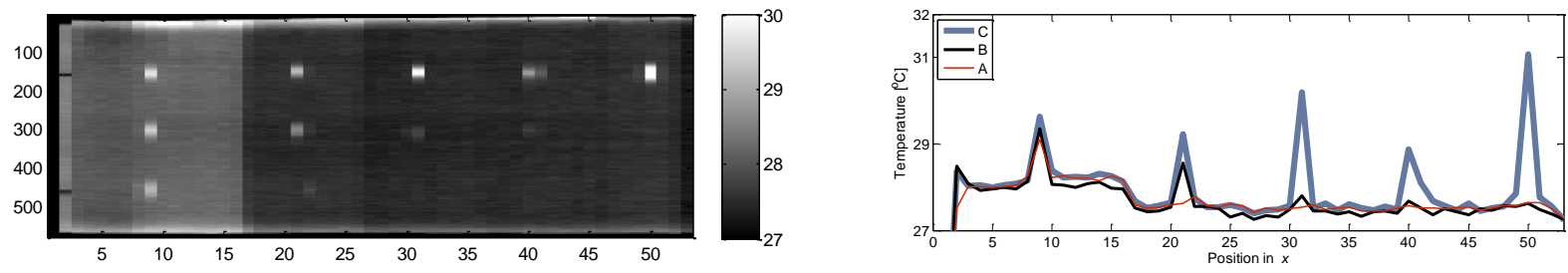

$v=30 \mathrm{~mm} / \mathrm{s}, f_{\text {rate }}=3.5 \mathrm{~Hz}, \mathrm{~N}=52$ frames, $t^{\prime}=3 \mathrm{~s}$

Fig. 6. The impact of the acquisition rate on temporal resolution after reconstruction.

Finally, the effect of applying advanced signal processing can be assessed by analysing the images presented in Fig. 7. The pseudo static sequence was processed by principal component thermography (PCT). The reconstructed thermogram in Fig. 7a correspond to $t^{\prime}=7.9 \mathrm{~s}$ for a scan inspection at $5 \mathrm{~mm} / \mathrm{s}$. PCT was applied over selected regions of interest (ROI). Two results are presented. The first one in Fig. $7 \mathrm{~b}$ cover a region of 9 defects (C3 to C5, B3 to B5, and A3 to A5) and shows a considerable improving in defect contrast for almost all defects, with the exception of defect A5 (the deepest one, $D / z=1.7)$, which is not detected even after processing. The second result in Fig. 7c, covers an area corresponding to a single defect (A4), and shows a further improvement in defect contrast: $S N R_{\text {raw }}(A 4)=6.1 \mathrm{~dB} v s$. $\mathrm{SNR}_{\mathrm{pct}}(\mathrm{A} 4)=12.2 \mathrm{~dB}$. 


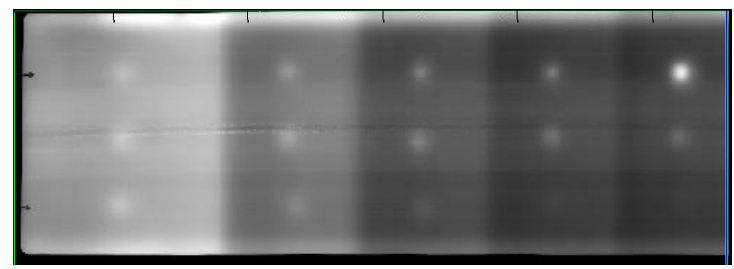

(a)

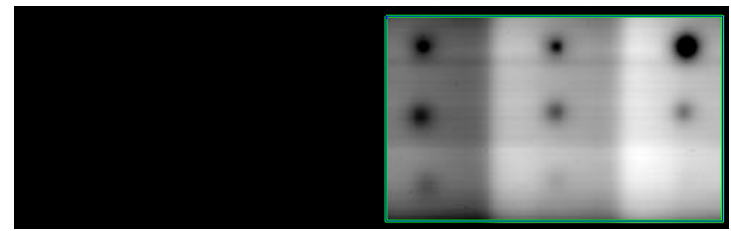

(b)

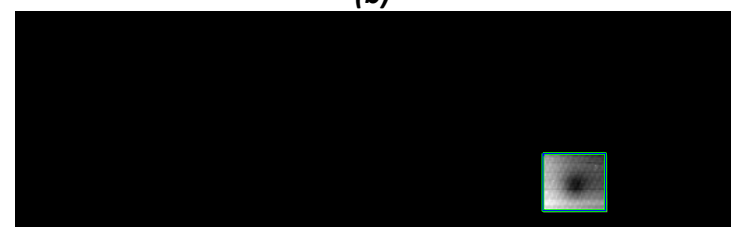

(c)

Fig. 7. The impact of advanced signal processing with principal component thermography $\left(\mathrm{v}=5 \mathrm{~mm} / \mathrm{s}, \mathrm{f}_{\mathrm{rate}}=6 \mathrm{~Hz}\right.$, $N=533$ frames, $\left.t_{o b s}=18.3 \mathrm{~s}, t^{\prime}=7.9 \mathrm{~s}\right)$ : (a) Raw reconstructed thermogram at time $t^{\prime \prime}=7.9 \mathrm{~s}$; (b) PCT (EOF ( $_{3}$ over a selected ROI covering 9 defects (C3 to C5, B3 to B5, and A3 to A5); (c) PCT (EOF $)$ over a selected ROI covering 1 defect (A4).

\section{Future work}

The next step of the research is to conduct a series of complete scans of the panel using a robotic arm to optimize the scanning speed together with a high-power heating source, and to perform advanced signal processing for defect characterization. Defect detectability will be compared through signal-to-noise calculations over all defects.

\section{References}

[1] Deonna F. Woolard and K. Elliott Cramer, "Line Scan Versus Flash Thermography: Comparative Study on Reinforced Carbon-Carbon," Proc. SPIE, Thermosense XXVII, G. Raymond Peacock, Douglas D. Burleigh and Jonathan J. Miles (eds.), 5782:315-323, 2005.

[2] Oswald-Tranta, B. and Shepard, S. "Comparison of Pulse Phase and Thermographic Signal Reconstruction Processing Methods," Proc. SPIE, Thermosense XXXV, Gregory R. Stockton, Fred P. Colbert, (eds.), 8705:87050S1-9, 2013.

[3] Deonna F. Woolard and K. Elliott Cramer, "The Thermal Photocopier: A New Concept for Thermal NDT," Proc. SPIE, Thermosense XXVI, Douglas D. Burleigh, K. Elliott Cramer and G. Raymond Peacock (eds.), 5405:366373, 2004.

[4] Gruss C, Balageas D. Theoretical and experimental applications of the flying spot camera. Proceedings of QIRT; 1992; pp. 19-24. QIRT Open Archives available from: http://qirt.gel.ulaval.ca/dynamique/index.php?idD=55; paper QIRT 1992-004.

[5] Oswald-Tranta, B. and Sorger, M. "Scanning pulse phase thermography with line heating," Quantitative InfraRed Thermography Jolurnal, 9(2):103-122, 2013. 